Zubayda M. Ibraheem

\title{
On Local Rings
}

zubaida_almulla@yahoo.com

Department of Mathematics,

College of Computer Science and Mathematics

University of Mosul, IRAQ

\section{Received on: $27 / 3 / 2013$}

\section{ABSTRACT}

Accepted on: 24/6/2013

A ring $\mathrm{R}$ is called local ring if it has exactly one maximal ideal. In this paper, we introduce some characterization and basic properties of this ring. Also, we studied the relation between local rings and Von Neumann regular rings and strongly regular rings.

Keywords: local, Von Neumann regular and strongly regular rings
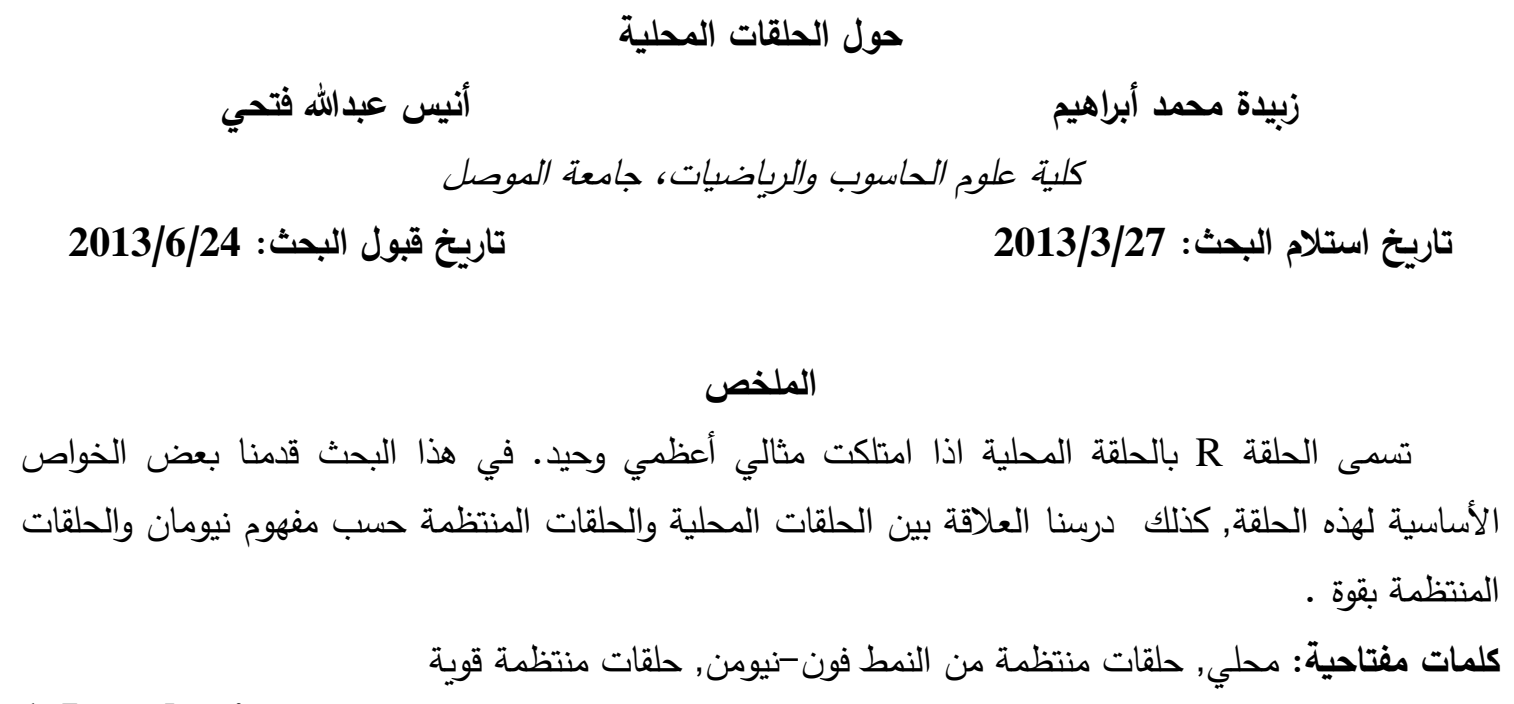

\section{Introduction}

Throughout this paper, $\mathrm{R}$ denotes associative rings with identity; all modules are unitary. For a subset $X$ of $R$, the right (left) annihilator of $X$ is denoted by $r(X)(1(X))$. If $X=\{a\}$, we usually abbreviate it to $r(a)(l(a))$. We write $J(R), Y(R), N(R)$ and $U(R)$ for the Jacodson radical, right singular ideal, the set of all nilpotent elements of an $\mathrm{R}$ and the set of all invertible elements of an $\mathrm{R}$ respectively. A right $\mathrm{R}-$ module $\mathrm{M}$ is called $\mathrm{P}$-injective, if for any principal right ideal $\mathrm{aR}$ of an $\mathrm{R}$ and any right $\mathrm{R}$-homomorphism of an $\mathrm{aR}$ into an $\mathrm{M}$ can be extended to one of an $\mathrm{R}$ into an $\mathrm{M}$. The ring $\mathrm{R}$ is called right $\mathrm{P}$-injective if an $R_{R}$ is $\mathrm{P}$-injective [8]. An ideal $\mathrm{I}$ of a ring $\mathrm{R}$ is said to be essential if and only if $I$ has a non-zero intersection with every non-zero ideal of an $R$. A ring $R$ is reduced if $N(R)=0$. A ring $R$ is said to be Von Neumann regular (or just regular) if and only if for each $a$ in $R$ there exists $b$ in $R$ such that $a=a b a$ [6]. A ring $\mathrm{R}$ is said to be right (left) quasi duo ring if every right (left) maximal ideal in $\mathrm{R}$ is right (left) ideal [ 7].

\section{The Local Rings:}

This section is devoted to give the definition of local rings with some of its chacterization and basic properties.

2.1. Definition [3] : A ring $R$ is said to be a local ring ,if any one of the following conditions holds:

1) $R$ has a unique maximal right ideal.

2) $R$ has a unique maximal left ideal. 
3) $R / J(R)$ is a division ring.

4) $R \backslash U(R)$ is an ideal of $R$ (all non-invertible elements of $R$ form a proper ideal ) i.e. $J(R)$ is the set of all non-invertible elements of $R$.

5) $\mathrm{R} \backslash \mathrm{U}(\mathrm{R})$ is a group under addition.

6) For any $n, a_{1}+a_{2}+\ldots+a_{n} \in U(R)$ implies that some $a_{i} \in U(R)$

7) $a+b \in U(R)$ implies that $a \in U(R)$ or $b \in U(R)$.

2.2. Proposition [1] : For any non-zero ring $R$, the following statements are equivalent

1) $R$ is a local ring.

2) if $a \in R$, then either a or $1-a$ is invertible.

Hazeinkel and Gubareni in [1] proved the following result:

2.3. Lemma [2] : Let $\mathrm{R}$ be a ring, all of whose non-invertible elements are nilpotent, then, $\mathrm{R}$ is a local ring.

As a consequence of this result and by using Proposition(2.3), we obtain the following result.

2.4. Proposition : Let an $\mathrm{R}$ be a local ring. Then, every element in an $\mathrm{R}$ is either invertible or nilpotent.

Proof : Suppose that an $\mathrm{R}$ is a local ring and let a be a non-invertible element in $\mathrm{R}$, then by Proposition (2.2), $1-\mathrm{a}$ is invertible, that is there exists $u$ in $R$ such that $(1-a) u=1$, and that can be held when $U=1+a+a^{2}+\ldots+a^{n-1} \in R$ and $(1-a)\left(1+a+a^{2}+\ldots+a^{n-1}\right)=1$, but $1-\mathrm{a}^{\mathrm{n}}=(1-\mathrm{a})\left(1+\mathrm{a}+\mathrm{a}^{2}+\ldots+\mathrm{a}^{\mathrm{n}-1}\right)=1$. Hence, $\mathrm{a}^{\mathrm{n}}=0$ and therefore, $\mathrm{a}$ is nilpotent element

2 .5. Corollary : Let an $R$ be a local ring. Then, $N(R)=J(R)$.

Proof : Let $0 \neq a \in J(R)$. Then, $a$ is non-invertible element. Thus, by Proposition (2.4) $a$ is nilpotent element, that is a $\in \mathrm{N}(\mathrm{R})$ Therefore, $\mathrm{J}(\mathrm{R}) \subset \mathrm{N}(\mathrm{R})$. It is clear that $\mathrm{N}(\mathrm{R}) \subset \mathrm{J}(\mathrm{R})[5]$. Therefore, $\mathrm{N}(\mathrm{R})=\mathrm{J}(\mathrm{R})$

Remark: If an $\mathrm{R}$ is a local ring, then $\mathrm{J}(\mathrm{R})$ is nilideal.

In the next proposition, we give the necessary condition for $\mathrm{R} / \mathrm{I}$ to be a local ring.

2.6. Proposition : Let $R$ be a commutative ring and $I$ be a primary ideal. Then, $R / I$ is a local ring.

Proof : Suppose that $\overline{\mathrm{a}}=(\mathrm{a}+\mathrm{I})$ is for every a $\in \mathrm{R}$. To prove that $\mathrm{R} / \mathrm{I}$ is a local ring. It is enough to show that R/I has exactly two idempotent elements which are $\overline{0}$ and $\overline{1}$ [3]. Let $a \in R$ such that $\bar{a}$ be a non-zero idempotent element of $R / I$, we have $a^{2}-a \in I$. Since $\mathrm{I}$ is a primary ideal of an $\mathrm{R}$ and a $\notin \mathrm{I}$, then there exists a non-negative integer $\mathrm{n}$ such that $(\mathrm{a}-1)^{\mathrm{n}} \in \mathrm{I}$. By the binomial theorem (which is valid in any commutative ring), $(\mathrm{a}-1)^{\mathrm{n}}=\sum_{k=0}^{n}(-1)^{\mathrm{n}-\mathrm{k}}\left(\begin{array}{l}n \\ k\end{array}\right) \mathrm{a}^{\mathrm{k}} \in \mathrm{I}$. Now, we prove by mathematical induction; first we claim that for each $\mathrm{k} \geq 2, \mathrm{a}^{\mathrm{k}}=\mathrm{a}+\mathrm{x}\left(1+\mathrm{a}+\ldots+\mathrm{a}^{\mathrm{k}-2}\right)$. Indeed; it is certainly true for $\mathrm{k}=2$, that is $\mathrm{a}^{2}=\mathrm{a}+\mathrm{x}$. Now, suppose the statement is true for $\mathrm{k}$, then we get the following equalities $a^{k+1}=a^{2}+x\left(a+a^{2}+\ldots+a^{k-1}\right)=a+x\left(1+a+\ldots+a^{k-2}\right)$ we conclude that for each non-negative integers $\mathrm{n}$, there is some $\mathrm{x}_{\mathrm{k}} \in \mathrm{I}$ such that $\mathrm{a}^{\mathrm{k}}=\mathrm{a}+\mathrm{x}_{\mathrm{k}}$. Now, $(-1)^{\mathrm{n}}$ $.1+\sum_{\mathrm{k}=1}^{\mathrm{n}}(-1)^{\mathrm{n}-\mathrm{k}}\left(\begin{array}{l}\mathrm{n} \\ \mathrm{k}\end{array}\right)\left(\mathrm{a}+\mathrm{x}_{\mathrm{k}}\right) \in \mathrm{I}$. But, $\quad(-1)^{\mathrm{n}} \cdot 1+\sum_{\mathrm{k}=1}^{\mathrm{n}}(-1)^{\mathrm{n}-\mathrm{k}}\left(\begin{array}{l}\mathrm{n} \\ \mathrm{k}\end{array}\right) \mathrm{a}=(-1)^{\mathrm{n}}(1-$ a). Hence, $1-\mathrm{a} \in \mathrm{I}$, and so $\overline{\mathrm{a}}=\overline{1}$. Therefore, $R / \mathrm{I}$ is a local ring

Example : In $(\mathrm{Z},+,),. 8 \mathrm{Z}$ is a primary ideal and $\mathrm{Z} / 8 \mathrm{Z}$ is a local ring, where $\mathrm{Z} / 8 \mathrm{Z}=\mathrm{Z}_{8}$ and has $2+8 Z$ as an unique maximal ideal .

2.7. Lemma [5] : For any ring $\mathrm{R}$ the followings are equivalent: 
1) $R$ has an unique prime ideal.

2) $R$ is a local ring and $J(R)$ is the intersection of all prime ideals of $R$.

3) Every non invertible element is nilpotent.

Now, we can obtain the following result

2.8. Proposition: If an $\mathrm{R}$ is a commutative ring and has exactly one prime ideal, then $\mathrm{R} / \mathrm{N}(\mathrm{R})$ is field .

Proof: Suppose an R has exactly one prime ideal, then by Lemma (2.7), $\mathrm{R}$ is a local ring and, hence $R / J(R)$ is a division ring and by Proposition (2.5), we have $J(R)=N(R)$ and since $R$ is commutative therefore $R / N(R)$ is field

Now, to show the relation between a local ring and Noetherain ring we have the following result from [1].

2.9. Lemma [1] : Suppose $\left\{I_{i}: i \in N\right\}$ is a family of proper right ideals of a ring $R$ with property that $I_{n} \subset I_{n+1}$ for all $n \in N$. Then, $I=U_{n \in N} I_{n}$ is proper right ideal

2.10. Lemma [1] : Any proper right ideal $I$ of a ring $R$ with identity is contained in a maximal proper right ideal.

2.11. Proposition: Let an $R$ be a local ring, then $R$ is a Noetherain ring .

Proof: Let $\left\{I_{i}: i \in N\right\}$ be a family of proper ideals in the ring $R$. Since, an $R$ is a local ring, then an $\mathrm{R}$ has a unique maximal ideal $\mathrm{M}$. Then, by Lemma (2.10), the maximal ideal $M$ contains all proper ideals in an $R$ that is $I_{i} \subseteq M$, for all $i \in N$. Hence, $\bigcup_{i=1}^{n} I_{i}$ $=I_{n}=M=J(R)$. Now, if $a \in I_{i}$ and $a \notin I_{i+1}$, then $I_{i} \not \subset I_{i+1} . B y \operatorname{Lemma}(2.9)$ we get $U_{i=1}^{n} I_{i} \neq M$. which is contradiction. Hence, for all $a \in I_{i}$ we have a $\in I_{i+1}$ thus $I_{i} \subseteq$ $\mathrm{I}_{\mathrm{i}+1}$, for all $\mathrm{i} \in \mathrm{N}$. That is $\mathrm{I}_{1} \subseteq \mathrm{I}_{2} \subseteq \mathrm{I}_{3} \subseteq \ldots \subseteq \mathrm{I}_{\mathrm{n}}$. Hence, an $\mathrm{R}$ satisfies (a.c.c.). Therefore, $\mathrm{R}$ is a Noetherain ring

Note: The converse of the above proposition is not true, by the following example :

Example : $(Z,+,$.$) , where Z$ is the set of integer number and $(Z,+,$.$) is a Noetherain$ ring, but not a local ring.

2.12. Proposition [1] : For any non-zero ring $R$ the following statements are equivalent

1) $R$ has a unique maximal right ideal.

2) $R$ has a unique maximal left ideal.

2.13. Proposition : Let an $R$ be a local ring. Then, $R$ is quasi duo ring.

Proof: Let an $\mathrm{R}$ be a local ring. Then, an $\mathrm{R}$ has unique maximal ideal $\mathrm{J}(\mathrm{R})$. Now by Proposition (2.12), $\mathrm{J}(\mathrm{R})$ is two sided ideal. That is every right (left) maximal ideal in an $\mathrm{R}$ is ideal. Hence, an $\mathrm{R}$ is quasi duo ring

Note :The converse of the proposition (2.13) is not true.

Example: Let an $\mathrm{R}=\left\{\left[\begin{array}{ll}a & b \\ 0 & c\end{array}\right]:\right.$ a,b,c $\left.\in \mathrm{Z}_{2}\right\}$ an $\mathrm{R}$ is quasi duo ring, but not a local ring.

\section{The Local Ring without Zero Divisor Element}

In this section, we give some results about the local ring without zero-divisor and some relation with other rings like division ring, Von Neumann regular, strongly regular and uniform rings.

3.1. Proposition: Let an $\mathrm{R}$ be a local ring. Then, every non-zero divisor element of $\mathrm{R}$ is a right invertible. 
Proof: Let $0 \neq \mathrm{a}$ be a non zero-divisor element in $\mathrm{R}$. Since, an $\mathrm{R}$ is a local ring, then either $\mathrm{aR}=\mathrm{R}$ or $\mathrm{aR} \neq \mathrm{R}$. If $\mathrm{aR}=\mathrm{R}$ then, there exists $\mathrm{b}$ in $\mathrm{R}$ such that $\mathrm{ab}=1$. Therefore, $\mathrm{a}$ is a right invertible. Now, if $a R \neq R$, then there exists a maximal right ideal $M$ such that $a R \subset M$. Since, an $R$ is a local ring, then $a R \subset M=J(R)$, which implies that a $\in J(R)$ and, hence by Proposition (2.5) a is nilpotent element. Therefore, $\mathrm{a}=0$ which is a contradiction, therefore $\mathrm{aR}=\mathrm{R}$ for all $\mathrm{a}$ in $\mathrm{R}$ and, hence a is invertible element

3.2. Proposition: Let $R$ be a local ring without zero-divisor. Then $R$ is regular ring.

Proof: Since, an $R$ is a local ring, then either $a R=R$, that is there exists $b \in R$ such that $a b=1$. Hence, $a^{2} b=a$. Thus, an $R$ is a strongly regular ring and therefore, an $R$ is $a$ regular ring. Now, if $\mathrm{aR} \neq \mathrm{R}$, then by the same method of the proof of proposition (3.1) we have $a=0$

3.3. Proposition:[2] For any ring $R$ the followings are equivalent :

1) $R$ is Von Neumann regular ring.

2) Every $R$-module is $P$-injective.

3) Every cyclic R-module is $\mathrm{P}$-injective.

3.4. Proposition:[4] If an $R$ is right $P$-injective, then $J(R)=Y(R)$.

Now, we give the main result of this section:

3.5. Proposition: Let an $\mathrm{R}$ be a local ring and without zero divisor. Then

1) $Y(R)=J(R)$.

2) $Y(R)=N(R)$.

proof : 1)Since, an $\mathrm{R}$ is a local ring and without zero divisor, then by Proposition (3.2) and Proposition (3.3) $\mathrm{R}$ is right $\mathrm{P}$-injective module and, hence by Proposition (3.4), we have $\mathrm{Y}(\mathrm{R})=\mathrm{J}(\mathrm{R})$

2) The proof is obvious

Finally, we give the following result :

3.6. Proposition: Let an $R$ be a ring and without zero-divisor. Then, $a R+r(a)=R$ if and only if $\mathrm{R}$ is a local ring.

Proof : Assume that an $\mathrm{R}$ is a local ring, then either $\mathrm{aR}=\mathrm{R}$ for any element $0 \neq \mathrm{a}$ in $\mathrm{R}$ so $\operatorname{ar}=1$, where $r \in R$ that is $a^{2} r=a$. Thus, $a(1-a r)=0$ implies that $(1-a r) \in r(a)$. Hence, $1=a r+(1-a r) \in a R+r(a)$. Therefore, $R=a R+r(a)$. Now, if $a R \neq R$, then by the same proof Proposition (3.1), $a=0$. Now, suppose that $R=a R+r(a)$, then there exists $r \in R, b \in r(a)$ such that $a r+b=1$ implies that $a^{2} r=a$, then $(1-a r) \in r(a)=0$, that is $1=a r$, thus a is right invertible. Now, since $1=a r$. Then, $a=a r a$ implies that $a-a r a=0$, hence (1-ra) $\in$ $r(a)=0$.Thus, $a$ is left invertible. Therefore, $a$ is a invertible .Now, we must prove that $(1-a)$ is not invertible. If (1-a) is invertible, that is $a \in J(R)$ and by the same method of the proof of Proposition (3.1) $a=0$ and this is contradiction, since $a \neq 0$. Thus, ( $1-a)$ is not invertible and, therefore by Proposition(2.2), an $\mathrm{R}$ is a local ring 


\section{REFERENCES}

[1] Hazeinkel, M., Gubareni, N. and Kirichenlo, V.V. (2004), "Algebars, Rings and Modules" Vol. 1 kluwer Academic publishers.

[2] Ibraheem Z.M. (1991), "On P-injective modules" M.Sc. Thesis, Mosul University.

[3] Lam, T.Y., (1991), "A First Course in Noncommutative Rings" Springerverlag New York, Inc.

[4] Nicholson, W. K. and Yousif M.F. (1995), "Principally Injective Rings", J. Algebra, 174, PP. 77-93.

[5] Naoum F.S. (2004), " On Semi Commutative $\pi$-Regular Rings", Ph.D. Thesis, Mosul University.

[6] Von Neumann, J. (1936), "On Regular rings", Proc. Nat. scad. Science U. S. A., Vol. 22, PP. 707-713.

[7] Yu, H.P., (1995), "On quasi duo ring", Glasgow Math. 37, PP. 21-31.

[8] Yue Chi Ming, R. (1974), "On Von Neumann regular rings", Proc, E Edinburgh Math. Soc. 19, PP. 89-91. 